\title{
Surface Hydrophobicity and Surface Rigidity Induce Spore Germination in Colletotrichum graminicola
}

\author{
J. Chaky, K. Anderson, M. Moss, and L. Vaillancourt
}

First and fourth authors: Department of Plant Pathology, Agricultural Science Center - North, University of Kentucky, Lexington 40546; and second and third authors: Department of Chemical and Materials Engineering, Anderson Hall, University of Kentucky, Lexington 40546. Current address of J. Chaky: Department of Plant Pathology, 448 Plant Sciences Hall, University of Nebraska, Lincoln 68583. Current address of M. Moss: Department of Research, 4500 San Pablo Road, Mayo Clinic Jacksonville, Jacksonville, FL 32224. Accepted for publication 20 February 2001.

\begin{abstract}
Chaky, J., Anderson, K., Moss, M., and Vaillancourt, L. 2001. Surface hydrophobicity and surface rigidity induce spore germination in Colletotrichum graminicola. Phytopathology 91:558-564.

We investigated the relationship between physical characteristics of artificial surfaces, spore attachment, and spore germination in Colletotrichum graminicola. Surface hydrophobicity and surface rigidity were

substrate for spore germination. Spore attachment was typically stronger to more hydrophobic surfaces, but certain hydrophilic surfaces also proved to be good substrates for spore attachment. In contrast to spore germination, appressorial induction was more dependent on attachment to a rigid substrate than it was on surface hydrophobicity. Appressoria were induced efficiently on hydrophilic surfaces, as long as there was significant conidial attachment to those surfaces.
\end{abstract} both signals for breaking dormancy and initiating spore germination, but spore attachment alone was not an important inducing signal. The presence of a carbon source overrode the necessity for a rigid, hydrophobic
Additional keywords: corn anthracnose, Glomerella graminicola.
Spore germination is a critical event in the life cycles of most fungi, and so it is an important potential target for disease control. Although various environmental and physiological factors affecting fungal spore germination have been investigated in a number of species, the signals and molecular controls involved in the breaking of fungal spore dormancy are not well understood (6). Our long-term goal is to elucidate these processes in the plant pathogenic fungus Colletotrichum graminicola (Ces.) G. W. Wils. (teleomorph $=$ Glomerella graminicola Politis).

C. graminicola causes anthracnose leaf blight and stalk rot of corn and, like most plant-pathogenic fungi, it produces multitudes of asexual conidia that are responsible for pathogen dispersal (2). For a conidium to initiate disease it must attach, germinate, and penetrate the surface of a susceptible corn plant. C. graminicola conidial attachment to corn leaf surfaces has been described (21). The conidia release an extracellular matrix when they become hydrated and are incubated on a leaf surface (21-23). This matrix contains glycoproteins and is involved in conidial attachment. $C$. graminicola germlings produce melanized appressorial cells that function as staging areas for mechanical and enzymatic penetration of the host cell $(2,4,28)$. Conidia of $C$. graminicola attach efficiently to many artificial surfaces (23), allowing investigation of the entire process of conidial attachment, germination, and appressorial formation in vitro.

Preliminary observations in our laboratory indicated that the efficiency of spore germination in $C$. graminicola varied significantly when spores were deposited onto different artificial surfaces. Thus, spores germinated at a high rate $(>80 \%)$ when they were deposited in water on a hydrophobic polystyrene petri dish,

Corresponding author: L. Vaillancourt; E-mail address: vaillan@pop.uky.edu

Publication no. P-2001-0320-01R

This article is in the public domain and not copyrightable. It may be freely reprinted with customary crediting of the source. The American Phytopathological Society, 2001. but spores deposited on a hydrophilic glass slide surface exhibited a comparatively poor germination rate $(<20 \%)$.

Attachment of spores to hydrophilic surfaces was reported to be relatively weak in comparison with attachment to less wettable, hydrophobic surfaces (23). Therefore, we hypothesized that strong attachment to a surface might be an important signal for induction of germination in $C$. graminicola. Attachment as a prerequisite for germination has been demonstrated in the plant-pathogenic fungus Phyllosticta ampelicida $(15,32,33)$ and in two aquatic hyphomycetes, Anguillospora longissima and Lunulospora curvula (38). To test the hypothesis that attachment to a surface was an inducing signal for spore germination in $C$. graminicola, we measured both attachment and germination of spores on a variety of surfaces exhibiting a range of surface wettabilities. To provide a frame of reference for our work, we compared the induction of spore germination in response to surface cues with the induction of appressoria in response to those same cues. Appressoria in $C$. graminicola and in other fungi are induced by attachment of the germ tube tip to a firm, hydrophobic surface (4).

\section{MATERIALS AND METHODS}

Fungal culture and production of conidia. We found that preparation of conidia for germination assays according to very strict protocols was critical to ensure consistent results. C. graminicola strain M1.001 was grown on potato dextrose agar (PDA) (Difco Laboratories, Detroit) under continuous fluorescent light. Conidial stock cultures were stored at $-80^{\circ} \mathrm{C}$ in $7.5 \%$ skim milk on silica (36), and cultures were started from this stock every 2 weeks to ensure consistent conidial production and quality for all experiments. Disposable sterile plastic pipette tips, test tubes, and microfuge tubes were used for all experiments. For each experiment, falcate conidia were harvested from point-inoculated cultures started from the silica cultures. Conidia were collected by flooding 12- to 15-day-old cultures with $10 \mathrm{ml}$ of sterile distilled deionized water $\left(\mathrm{ddH}_{2} \mathrm{O}\right)$ (NANOpure system, Barnstead, Boston) 
and gently scraping the surface with a sterile loop. The suspension was filtered through cheesecloth, sterile $\mathrm{ddH}_{2} \mathrm{O}$ was added to a final volume of $50 \mathrm{ml}$, and the conidial suspension was centrifuged at $2,000 \times g$ for $5 \mathrm{~min}$ at $20^{\circ} \mathrm{C}$. The spores were washed three times in sterile $\mathrm{ddH}_{2} \mathrm{O}$ to remove the spore mucilage and its associated germination inhibitor (20), and they were finally resuspended in sterile $\mathrm{ddH}_{2} \mathrm{O}$, at a concentration of $1 \times 10^{4}$ spores per $\mathrm{ml}$. The spore suspensions were used immediately for experiments.

C. graminicola oval conidia (27) were used for some experiments. To produce oval conidia, falcate conidia were harvested from a single PDA plate containing a 2-week-old culture of M1.001. The spores were rinsed three times in sterile $\mathrm{ddH}_{2} \mathrm{O}$, then added to $150 \mathrm{ml}$ of liquid complete medium (19) in a $250-\mathrm{ml}$ flask. The flask was shaken at $200 \mathrm{rpm}$ overnight at $30^{\circ} \mathrm{C}$. The following day, the flask contained very few, if any, ungerminated falcate conidia, and large numbers of oval conidia. The oval conidia were collected by centrifugation, washed three times in sterile $\mathrm{ddH}_{2} \mathrm{O}$, and finally resuspended in sterile $\mathrm{ddH}_{2} \mathrm{O}$ at a concentration of $1 \times 10^{4}$ spores per $\mathrm{ml}$. The spore suspensions were used for experiments immediately.

Surfaces. Various firm surfaces exhibiting different inherent wettabilities were used to investigate conidial germination and attachment. The surfaces included: adaxial surface of a corn leaf (Zea mays L. var. Mo940), polystyrene petri dish (Becton Dickinson and Company, Franklin Lakes, NJ), polystyrene sheet (0.1 mm thick) (Wards Plastics, Rochester, NY), FEP Teflon (12.5 $\mu \mathrm{m}$ thick) (DuPont, Circleville, $\mathrm{OH})$, mylar $(25 \mu \mathrm{m}$ thick) (Dayton Plastics, Dayton, OH), plastic coverslips (Fisherbrand, Fisher Scientific Co., Pittsburgh), glass coverslips (Gold Seal, Becton Dickinson and Company, Franklin Lakes, NJ), glass slides (Gold Seal, Becton Dickinson and Company), Teflon-coated multiwell (MW) slides (Electron Microscopy Sciences, Ft. Washington, PA), and 4\% water agar (Difco). Plastic coverslips thickly coated with silicone vacuum grease (Beckman Coulter, Fullerton, CA) were also used in some spore germination assays.

The wettability of polystyrene sheets $(0.1 \mathrm{~mm}$ thick $)$ was modified by UV-irradiation as described by Kuo and Hoch (15). Following UV treatment, the sheets were rinsed three times with $\mathrm{ddH}_{2} \mathrm{O}$, air-dried, and stored in the dark until later use. The treated polystyrene sheets were cut into 2 -cm sections just before use in germination assays.

Contact angle measurements. The wettability of each surface was determined by measuring the advancing contact angles, $\theta_{\mathrm{A}}$, of water droplets on the surfaces $(30,40)$. A larger contact angle indicated a less wettable (more hydrophobic) surface.

Conidial germination. A spore suspension $\left(1 \times 10^{4}\right.$ spores per $\mathrm{ml})$ was deposited as three $100-\mu \mathrm{l}$ drops onto each of the test surfaces. A minimum of 100 spores was counted for each drop. The test surfaces were placed inside polystyrene petri dishes, and the petri dishes were placed into a humidity chamber. The inside bottom surface of the petri dish itself served as one of the test surfaces. The humidity chambers were placed inside an incubator at $23^{\circ} \mathrm{C}$ and spore germination was assessed after $24 \mathrm{~h}$. A conidium was recorded as germinated if it had produced one or more germ tubes, with or without appressoria. Each experiment was repeated three or four times.

Spore germination was also investigated in hanging drops. Spore suspensions were deposited in three 100- $\mu$ l drops onto the undersides of inverted MW slides that were balanced on two $1,000-\mu l$ pipette tips. The slide and the hanging drops were placed into a petri dish inside a humidity chamber and incubated for $24 \mathrm{~h}$. Hanging drops were then turned upright, and germination was assessed as described previously.

The corn inbred Mo940, which is highly susceptible to $C$. graminicola, was used for in vivo spore germination assays (26). These assays were conducted using microhumidity chambers, as described by Bergstrom and Nicholson (1). The youngest fully expanded leaf of a corn seedling at the V4 growth stage (29) was inoculated with $30 \mu \mathrm{l}$ of a spore suspension $\left(3.3 \times 10^{4}\right.$ spores per $\mathrm{ml})$ per well of the microhumidity chamber. Three leaves were included in each experiment, and the experiment was repeated three times. After $24 \mathrm{~h}$, spores on the leaf surface were stained with $0.02 \%$ Calcofluor (Polysciences, Inc., Warrington, PA) and at least 100 spores per inoculation site were assessed for germination using epifluorescence microscopy.

Conidial attachment. Attachment of the conidia to the test surfaces was measured with one of two different methods. For the first assay method, a parallel-plate flow chamber (Biomedical Engineering Laboratory, Rice University, Houston, TX) was used to apply a controlled, measurable shear stress to spores that had settled onto various surfaces. The procedure of Moss et al. (24) was used for all of these experiments. For each experiment, a spore suspension $\left(2.5 \times 10^{6}\right.$ spores per $\left.\mathrm{ml}\right)$ was introduced into the chamber, and spores were allowed to settle onto and attach to the experimental surface in the absence of shear for $30 \mathrm{~min}$. A solution of dextran (11.5\%, wt/vol) (Sigma Chemical Co., St. Louis) was then perfused through the chamber for $5 \mathrm{~min}$ at flow rates ( 4.5 to $6.7 \mathrm{ml} / \mathrm{min}$ ) that produced a shear stress in the range of 38 to 42 dynes per $\mathrm{cm}^{2}$. Experiments were viewed on a video monitor and the images were recorded on a video cassette recorder for later analysis. The final percent spore detachment after $5 \mathrm{~min}$ of applied shear force was calculated for each surface. The surfaces tested using this method included Teflon, mylar, polystyrene petri dishes, glass coverslips, glass slides, and MW glass slides. Plastic coverslips, 4\% agarose, and polystyrene sheeting could not be tested in the flow chamber for technical reasons.

The second procedure consisted of vortexing a surface with its adhered spores in water to remove spores that were not firmly attached. The force applied to the spores by vortexing, although reproducible, could not be directly measured by this method. Spore suspensions $\left(1 \times 10^{4}\right.$ spores per $\left.\mathrm{ml}\right)$ were deposited in $100-\mu l$ drops onto the surface. As a control, additional spore drops were deposited onto replicate surface samples. The spores were allowed to settle for $30 \mathrm{~min}$, after which the test surface was placed into a $150-\mathrm{ml}$ beaker with $30 \mathrm{ml}$ of $\mathrm{ddH}_{2} \mathrm{O}$. The surface was vortexed in the beaker for $30 \mathrm{sec}$ at speed setting 6 on a Vortex-Genie 2 (Model G-560; VWR Scientific, Scientific Industries, Inc., Bohemia, NY). Each treatment was tested three times in each experiment, and each experiment was replicated at least twice. The untreated controls were counted in the same manner, and percentage of attachment was calculated from the ratio between the experimental and control counts. This method was used to assess attachment in the concanavalin A (Con A) experiments.

Effect of added substances on spore attachment and germination. Nutrients were added to spore suspensions in some experiments. Nutrients tested included yeast extract (1\%, wt/vol); and all of the following compounds at a final concentration of 100 mM: glucose, sucrose, maltose, glutamic acid (monosodium salt), and ammonium nitrate.

Con A (Type IV) (Sigma) was added to spore suspensions in some experiments. Con A was dissolved in sterile $\mathrm{ddH}_{2} \mathrm{O}$ because buffer salts affected attachment of another Colletotrichum spp. $(23,39)$. Final concentration of Con A ranged from 0 to $1 \mathrm{mg} / \mathrm{ml}$. Con A was preincubated with the spores for $30 \mathrm{~min}$ prior to deposition of the spores onto the surface (23). In some experiments, glucose $\left(7.4 \times 10^{-5} \mathrm{M}\right)$ or methyl-D- $\alpha$-mannoside (up to $500 \mathrm{mM}$ ) was added to the Con $\mathrm{A} /$ spore suspensions. In the experiments with methyl-D- $\alpha$-mannoside, the treated and control spore suspensions were deposited immediately, as described by Kuo and Hoch (15), rather than after a 30-min preincubation period.

Experimental design and analysis of data. All experiments were repeated three to four times. The results of the individual 
experiments were pooled for statistical analysis. All values are represented in the figures and in the text as averages \pm the standard deviation. Correlation analyses and multiple comparison tests (Duncan's multiple range test) were conducted using the SAS statistical analysis package (SAS Institute Inc., Cary, NC).

\section{RESULTS}

Contact angle measurements. The degree of surface wettability was determined by measuring the advancing angle $\left(\theta_{\mathrm{A}}\right)$ at the three-phase interface of water, air, and surface (40). Advancing contact angles were recorded for sterile $\mathrm{dd}_{2} \mathrm{O}$ on all surfaces. The values ranged from a high of $133^{\circ}$ for a corn leaf to a low of $11^{\circ}$ for glass slides from the NH lot (Table 1).

The relationship between surface wettability, attachment, and germination. Spore germination rates were highest $(>70 \%)$ on surfaces with $\theta_{\mathrm{A}}$ values $>55^{\circ}$ including the corn leaf, Teflon, plastic coverslip, mylar, petri plate, and glass coverslip. Germination rates were significantly lower $(<20 \%)$ on the less hydrophobic, more wettable surfaces with $\theta_{\mathrm{A}}$ values $<50^{\circ}$ (Table 1 ). A significant positive correlation was observed between the germination rate and the surface contact angle $(r=0.810, P=0.0001)$. The relationship between the germination rate and the contact angle was not linear, but approximated a sigmoidal curve with the point of inflection at approximately $\theta=50^{\circ}$.

Germination of oval conidia on some surfaces was also evaluated. In contrast to falcate conidia, there was no significant relationship between the germination rate of oval conidia and surface wettability. Oval conidia germinated similarly, at a rate of $>85 \%$ on glass slides, plastic coverslips, glass coverslips, and polystyrene petri dishes (data not shown).

Detachment of falcate spores from seven of the surfaces was observed in the parallel-plate flow chamber (Fig. 1). As surface wettability increased, rates of spore detachment also generally increased. The percentage of spore detachment was highest for the glass slides from the NH lot, which also had the highest wettability value of the seven surfaces. However, the negative correlation between percent spore detachment and surface contact angle $(r=-0.738)$ was statistically not significant, $P=0.058$. Percentage of spore detachment and germination rates on these seven surfaces were rather weakly correlated $(r=-0.626, P=$ 0.0004).

The relationship between surface wettability, attachment, and appressorial induction. Appressorial induction was evaluated on each of the surfaces. There was no strong correlation between surface wettability and appressorial induction $(r=0.406$, $P=0.010)$, but there was a more significant correlation between the detachment of spores from the surfaces and appressorial induction $(r=-0.709, P=0.0001)$.

TABLE 1. Germination of Colletotrichum graminicola spores on surfaces with different wettabilities

\begin{tabular}{lcc}
\hline Surface & Wettability $^{\mathrm{a}}\left(\theta_{\mathrm{A}}\right)$ & ${\text { \% } \text { Germination }^{\mathrm{b}}}^{\mathrm{b}}$ \\
\hline Corn leaf & $133.4 \pm 2.4$ & $75.0 \pm 8.2$ \\
Teflon & $102.4 \pm 3.1$ & $87.5 \pm 6.0$ \\
Plastic coverslip & $91.0 \pm 1.7$ & $76.9 \pm 10.6$ \\
Mylar & $74.0 \pm 4.2$ & $79.5 \pm 10.0$ \\
Polystyrene petri dish & $71.8 \pm 2.5$ & $81.5 \pm 9.6$ \\
Glass coverslip & $58.1 \pm 2.2$ & $74.4 \pm 19.2$ \\
$4 \%$ agarose & $44.9 \pm 2.8$ & $11.6 \pm 10.0$ \\
Glass slide $\mathrm{PR}^{\mathrm{c}}$ & $20.3 \pm 2.0$ & $19.8 \pm 9.8$ \\
Glass slide $\mathrm{MW}^{\mathrm{d}}$ & $12.9 \pm 3.0$ & $2.2 \pm 3.0$ \\
Glass slide $\mathrm{NH}^{\mathrm{c}}$ & $11.6 \pm 3.1$ & $18.4 \pm 11.0$ \\
\hline
\end{tabular}

a $\theta_{\mathrm{A}}$, advancing angle.

b Germination was assessed after $24 \mathrm{~h}$ incubation.

c PR and NH refer to location where slide coating was applied, as indicated by lot number (Gold Seal, Becton Dickinson Labware).

d MW refers to Teflon-coated multiwell slides (Electron Microscopy Science).
The effect of nutrients on spore germination. Spores incubated in sterile $\mathrm{ddH}_{2} \mathrm{O}$ on a MW glass slide or in a hanging drop germinated very poorly $(0.1 \pm 0.2 \%$ on the MW glass slide, and $0.6 \pm 0.3 \%$ in the hanging drop). When $100 \mathrm{mM}$ glucose was added, spores germinated at a rate of $65.5 \pm 13.9 \%$ in the upright position and $42.3 \pm 9.2 \%$ in the hanging drop. A Student's $t$ test indicated that the difference in germination between the upright and hanging drops was not significant $(\alpha=0.05)$. Simple sources of nitrogen including glutamic acid and ammonium nitrate did not significantly increase germination on MW glass slides, whereas carbon sources including glucose, sucrose, and maltose increased germination to a much greater extent (Fig. 2). Yeast extract, a complex source of both carbon and nitrogen, had the greatest positive effect on germination on MW glass slides (Fig. 2).

Germ tubes produced in the presence of carbon were typically longer than those produced in plain water. A majority of spores that germinated in the presence of carbon on a firm surface produced an additional long germ tube (at least the length of the spore) without, or very rarely with, an appressorium, from the second cell of the spore. This second germination event was never observed in water or in the presence of simple nitrogen sources alone. Spores that germinated in hanging drops did not produce a second germ tube, and they never produced appressoria, either in the presence or absence of nutrients.

The effect of surface rigidity on spore germination and appressorial induction on a hydrophobic surface. Plastic coverslips and silicone grease possess similar wettability characteristics, but rates of spore germination differed significantly on these surfaces. A plastic coverslip is rigid, whereas silicone grease is comparatively soft. The rate of spore germination on plastic coverslips $\left(\theta_{\mathrm{A}}=91^{\circ}\right)$ was $96.3 \pm 2.0 \%$ in sterile $\mathrm{ddH}_{2} \mathrm{O}$, and $98.5 \pm 0.7 \%$ in glucose, and appressorial induction was $99.1 \pm$ $0.5 \%$ (water) and $98.3 \pm 1.2 \%$ (glucose). In contrast, the rate of spore germination on silicone grease-coated plastic coverslips $\theta_{\mathrm{A}}=99.9^{\circ}$ was only $13.2 \pm 7.1 \%$ in sterile $\mathrm{ddH}_{2} \mathrm{O}$, and this increased to $73.6 \pm 12.5 \%$ in the presence of $100 \mathrm{mM}$ glucose. Germinated spores on silicone, whether in $\mathrm{ddH}_{2} \mathrm{O}$ or glucose, never produced appressoria.

The effect of Con A on spore germination and attachment. Con $\mathrm{A}$ is a lectin that interferes with attachment of $C$. graminicola to hydrophobic surfaces (23). Con A was used to further test our hypothesis that the effect of surface wettability on spore germination was mediated through spore attachment to the surface. The percentage of spore attachment to plastic coverslips, measured by the vortex assay, was significantly reduced by the addition of $100 \mu \mathrm{g} / \mathrm{ml}$ or more of Con A (Fig. 3). However, germination was unaffected by the addition of Con A at any of the tested concentrations. Neither methyl-D- $\alpha$-mannoside nor glucose significantly increased spore attachment in the presence of $100 \mu \mathrm{g} / \mathrm{ml}$ or $1 \mathrm{mg} / \mathrm{ml}$ of Con A (data not shown).

Appressorial production was significantly reduced only at a concentration of $1 \mathrm{mg} / \mathrm{ml}$ of Con A, in which $27.1 \pm 13.2 \%$ of germinated spores produced appressoria, compared with $96.8 \pm$ $1.8 \%$ of germinated spores in the control. The addition of methyl$\mathrm{D}-\alpha$-mannoside or glucose also did not significantly increase appressorial induction in the presence of $1 \mathrm{mg} / \mathrm{ml} \mathrm{Con} \mathrm{A} \mathrm{(data} \mathrm{not}$ shown).

We measured the germination of spores deposited in $1 \mathrm{mg} / \mathrm{ml}$ of Con A under noninducing conditions to determine whether Con A alone stimulated the breaking of spore dormancy. Germination in $1 \mathrm{mg} / \mathrm{ml}$ Con A on MW glass slides $(24.2 \pm 7.6 \%)$ and in hanging drops $(26.5 \pm 7.1 \%)$ was significantly increased over the controls in $\mathrm{ddH}_{2} \mathrm{O}$ alone $(3 \pm 3.2 \% \mathrm{MW}$ slides, and $0.3 \pm 0.3 \%$ hanging drops). However, these germination rates were significantly lower than those observed in the presence of the same concentration of Con A on plastic coverslips. Concentrations of Con A lower than $1 \mathrm{mg} / \mathrm{ml}$ had no significant effect on spore germination in hanging drops or on MW glass slides (data not shown). 
UV-treated polystyrene. Based on the correlation we observed between surface wettability and spore germination in our initial experiments, we hypothesized that surface hydrophobicity is an important signal for induction of germination. The relationship between surface wettability and spore germination was further tested with UV-treated polystyrene sheets according to the method of Kuo and Hoch (15). The wettability characteristics of polystyrene were altered by exposure to UV light, as indicated by the measurement of contact angle (Fig. 4). The increase in wettability negatively affected spore germination on the polystyrene. Only $16.8 \pm 9.7 \%$ of spores germinated on polystyrene that had been treated at $2 \mathrm{~cm}$ from the light, compared with $93.5 \pm 0.1 \%$ germination on untreated polystyrene. A relatively small change in contact angle, from $54.3 \pm 5.5^{\circ}$ for polystyrene that had been $2 \mathrm{~cm}$ from the light to $65.1 \pm 3.7^{\circ}$ for polystyrene that had been $4 \mathrm{~cm}$ from the light, corresponded to a large increase in germination, from $16.8 \pm 9.7 \%$ to $78.9 \pm 5.3 \%$. The rate of spore germination was positively correlated with the surface contact angle $(r=$ $0.861)$ at $P=0.0001$.

Treatment of the polystyrene with UV also reduced appressorial induction. Only $29.9 \pm 26 \%$ of germinated spores produced appressoria on polystyrene that had been treated at $2 \mathrm{~cm}$ from the light compared with $98.5 \pm 0.1 \%$ on untreated polystyrene.

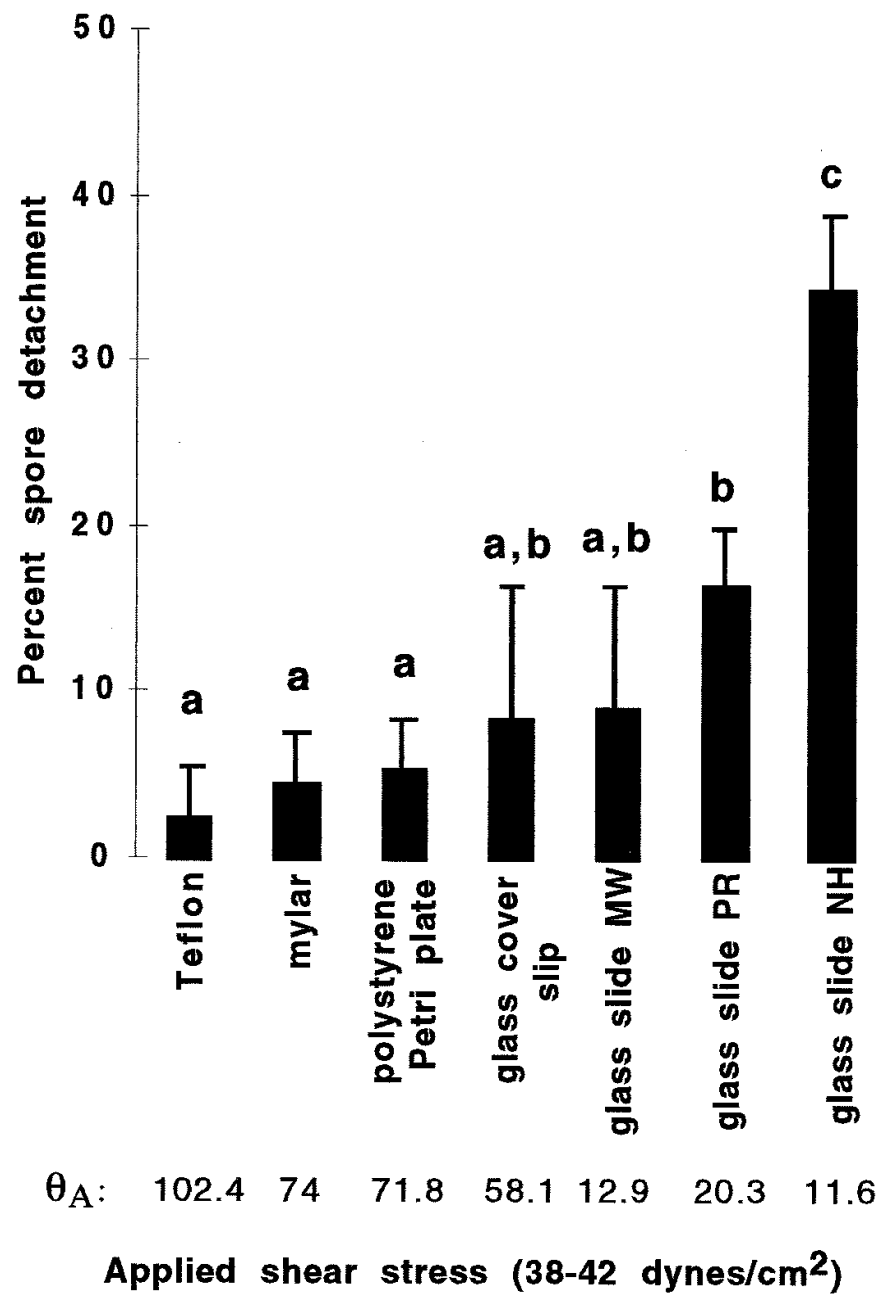

Fig. 1. Detachment of Colletotrichum graminicola spores from various surfaces under a controlled shear stress produced within the parallel-plate flow chamber. Surface wettability, measured as water contact angle, is indicated below each surface label. Percentage of spore detachment is indicated on the $y$ axis. Error bars represent the standard deviation. Columns labeled with the same letter are not significantly different according to Duncan's multiple range test $(P=0.05)$.
Appressorial induction on polystyrene treated at distances further than $2 \mathrm{~cm}$ from the light was not significantly reduced compared with the untreated control (data not shown).

\section{DISCUSSION}

Our goal in this study was to dissect and define physical cues that trigger spore germination of $C$. graminicola on certain artificial surfaces. We found that germination was efficiently induced by contact with a rigid, hydrophobic surface $\left(\theta_{\mathrm{A}}\right.$ greater than $\left.50^{\circ}\right)$. Although sufficient to trigger germination, contact was not necessary because germination occurred in its absence if a source of carbon was available. In contrast, attachment to a firm surface, whether hydrophobic or hydrophilic, appeared to be both sufficient and necessary for the induction of appressoria in $C$. graminicola.

A relationship between spore germination and surface hydrophobicity has been investigated in only a few other studies. Germination of Phyllosticta ampelicida pycnidiospores was found to be dependent on surface wettability characteristics, and spore germination was highest on the most hydrophobic surfaces (15, 32,33). In contrast to P. ampelicida and C. graminicola, spores of $C$. lagenarium germinated at a higher rate on hydrophilic surfaces

$\mathbf{a}$

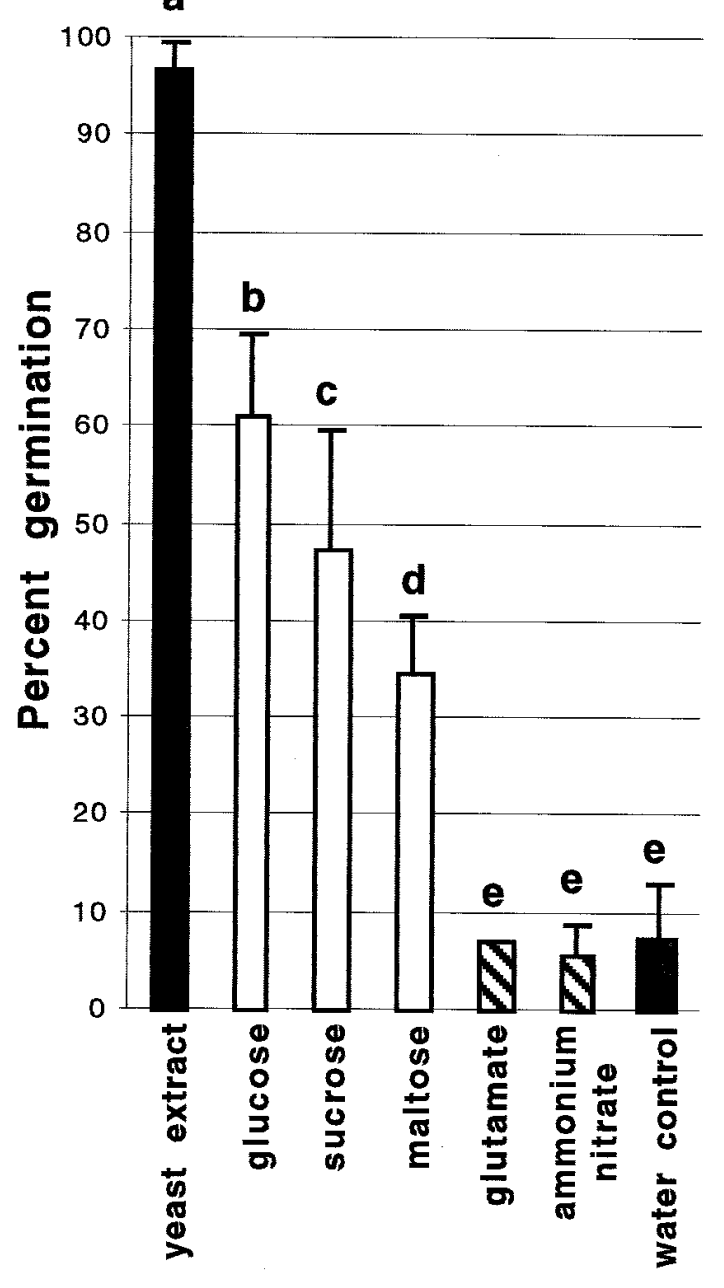

Treatment

Fig. 2. Effect of addition of nutrients on spore germination of Colletotrichum graminicola on multiwell glass slides. The nutrients include a complex carbon/nitrogen source ( $1 \%$ yeast extract, wt/vol) (filled bar), various carbon sources at $100 \mathrm{mM}$ (open bars), and various nitrogen sources at $100 \mathrm{mM}$ (hatched bars). A water control is included. Error bars indicate the standard deviation. Columns labeled with the same letter are not significantly different according to the Duncan's multiple range test $(P=0.05)$. 
than on hydrophobic ones (34). Other observations in that report, such as an ability to rescue spore germination on noninductive surfaces by addition of a nutrient source, suggest there may be an underlying similarity in the control of germination between $C$. lagenarium and $C$. graminicola.

In contrast to falcate conidia, oval conidia of $C$. graminicola germinated very efficiently on artificial surfaces in water regardless of surface hydrophobicity. This may reflect differences in biological function between these two spore types. Falcate conidia are produced in acervuli on the surfaces of plant tissues, and they are the primary infective propagule of $C$. graminicola. Oval conidia are produced only inside plant tissues during lesion development, and do not appear to have a role in plant-to-plant dissemination. Instead, they probably function to move the pathogen systemically within the plant $(2,27)$. Thus, surface hydrophobicity would not be expected to be an important signal for germination of oval conidia.

A previous study reported that the spores of $C$. trifolii germinate poorly in the absence of contact with a hard surface (37). Spore germination of $C$. truncatum was reported to increase with increasing concentrations of agar in the medium, suggesting that it may be quantitatively related to substrate firmness, but an effect of added nutrients from the agar cannot be eliminated from consideration (7). Our observations of spore germination on silicone grease also suggest that substrate firmness is important for optimal germination of $C$. graminicola spores in the absence of nutrients. It has been suggested that surface contact "primes" the conidia via a $\mathrm{Ca}^{2+}$-calmodulin-mediated signaling pathway, making the spores competent to respond to other germinationinducing signals (14).

An ability of fungal spores to attach to hydrophobic surfaces is common. Attachment is generally believed to function as a pathogenicity factor by preventing the spores from being displaced from a hydrophobic plant surface, and by facilitating the close contact between the spore and the host surface required for

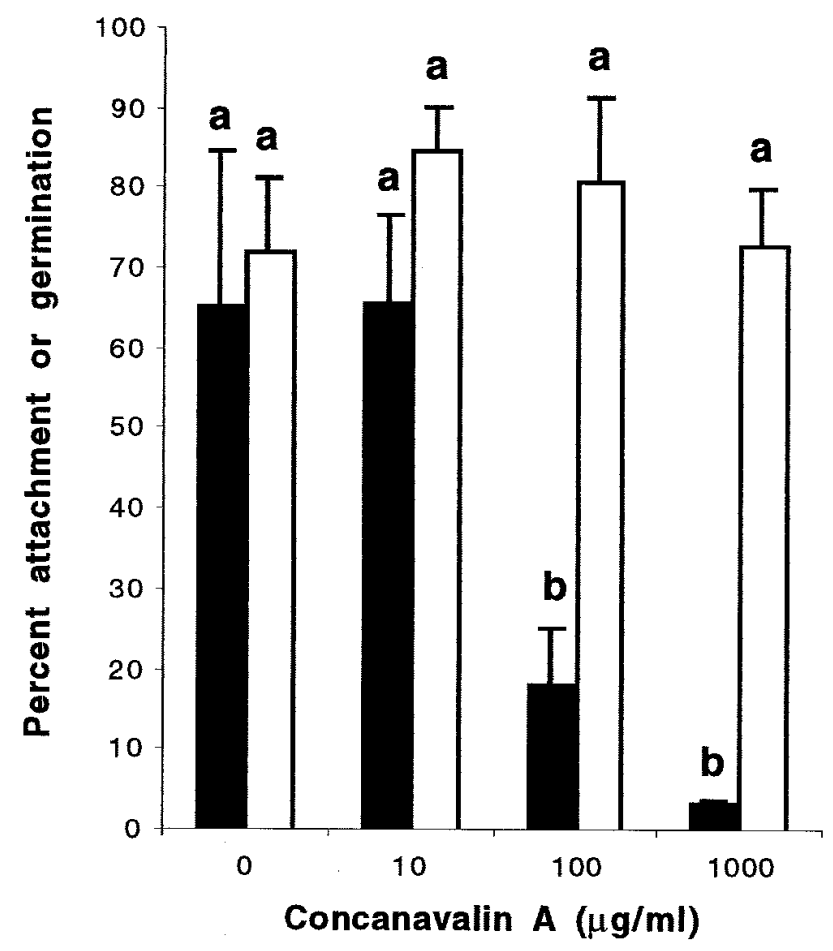

Fig. 3. Effect of Concanavalin A on attachment (filled bar) and germination (open bar) of Colletotrichum graminicola conidia on plastic coverslips. Percent attachment and germination are indicated on the $y$ axis. Error bars indicate the standard deviation. Columns in the same series (filled or open bars) labeled with the same letter are not significantly different according to Duncan's multiple range test $(P=0.05)$. host-pathogen signaling $(10,25)$. The relationship of fungal spore attachment to surface wettability has been investigated in a number of species. Spores of $C$. graminicola exhibited stronger attachment to less wettable polystyrene petri dishes than to more wettable glass slides (23). Conidia of Magnaporthe grisea, $C$. musae, and $P$. ampelicida, and urediospores of Uromyces appendiculatus, also attached more firmly to hydrophobic surfaces than to hydrophilic surfaces $(8,15,31,35)$. Conversely, conidia of Nectria haematococca and Cochliobolus heterostrophus exhibited nonspecific attachment to various surfaces, indicating no relationship between surface hydrophobicity and strength of attachment $(3,11,12)$.

We were able to confirm the report of Mercure et al. (23) by demonstrating that the strength of spore attachment in C. graminicola was related to surface hydrophobicity, and that attachment was generally strongest to the most hydrophobic surfaces. However, we also observed that factors other than hydrophobicity affected attachment in some cases. Spores attached strongly to the surfaces of some glass slides, despite their relatively high wettability values. Our observations of the behavior of the spores as they flowed through the chamber over these surfaces may help to explain this anomaly. Spores were frequently drawn out of the flowing spore suspension down onto these glass slide surfaces. This behavior was not commonly observed for the other surfaces. The attractive force between the spores and the surfaces in this case may be due to a charge interaction. Electromotive charge interactions are relatively strong. Kuo and Hoch (15) provided a similar explanation for the strong attachment of pycnidiospores of $P$. ampelicida to positively charged amine-terminated silanes

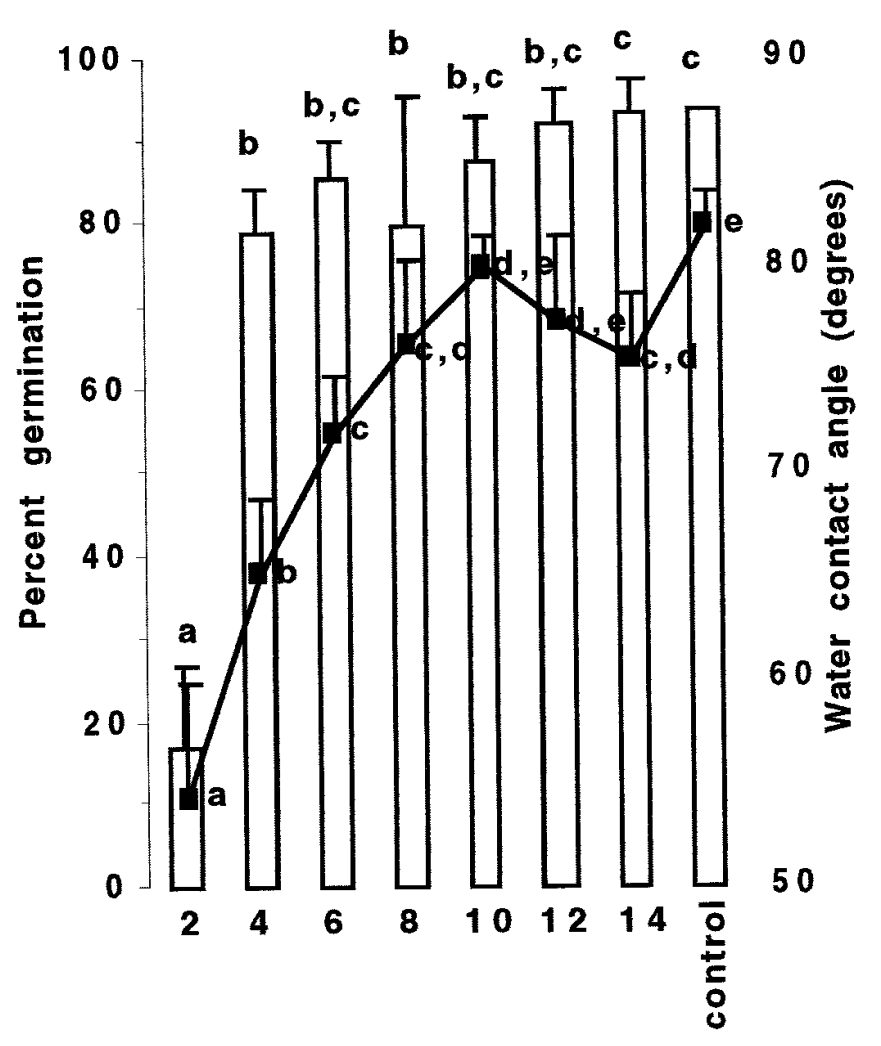

Distance from UV source $(\mathrm{cm})$

Fig. 4. Spore germination of Colletotrichum graminicola on UV-treated polystyrene. Spore germination (bars) was recorded after $24 \mathrm{~h}$, and contact angle (line) was measured following the procedure outlined. Untreated polystyrene was included in the assay. Percent germination is indicated on the primary $y$ axis, and water contact angle is on the secondary $y$ axis. Error bars indicate the standard deviation. Data points in the same series (bars or line) labeled with the same letter are not significantly different according to Duncan's multiple range test $(P=0.05)$. 
exhibiting hydrophilic characteristics and supporting poor germination rates. This result illustrates the value of being able to observe the behavior of the spores in the flow chamber as they approach and attach to various surfaces.

We further investigated the relationship between attachment and spore germination in experiments with the lectin Con A. Con A specifically binds $\alpha$-mannans and $\alpha$-glucans, and has been reported to interfere with fungal attachment in several species $(8$, 16-18,22,23,32). Attachment of spores of $C$. graminicola and $P$. ampelicida to polystyrene was completely inhibited, or reduced to $1 \%$, respectively, at a concentration of $1 \mathrm{mg} / \mathrm{ml}$ of Con A $(23,32)$. We found that $1 \mathrm{mg} / \mathrm{ml}$ of Con A reduced attachment of $C$. graminicola spores to plastic coverslips in the vortex assay from $65 \%$ to only $3 \%$. In previous reports, addition of haptans, including methyl-D- $\alpha$-mannoside, glucose, or mannose, reduced the negative effect of Con A on attachment, presumably through competition for carbohydrate binding sites $(23,32)$. Surprisingly, we were unable to reproduce this result. Adding glucose or methyl-D- $\alpha$-mannoside at the rates and in the manner suggested in the previous reports, or even at higher rates, did not increase spore attachment in the presence of Con A in our experiments. As well as binding to carbohydrates via specific, hydrophobic, carbohydrate binding sites, lectins also bind proteins or other substances at additional binding sites (13). Thus, our results could suggest that the interference with spore attachment by Con A in our experiments was mediated by binding to sites other than $\alpha$ mannans or $\alpha$-glucans. One possibility would be hydrophobic proteins, such as those described from the fibrillar, preformed spore coat of $C$. lindemuthianum (9). Monoclonal antibodies raised against two proteins found in this spore coat significantly reduced attachment of spores to polystyrene petri dishes. We agree with the suggestion of the authors of that study that attachment of spores to hydrophobic surfaces involves hydrophobic interactions between the surfaces and these preformed proteins.

Even though Con A significantly reduced spore attachment, germination was unaffected. This is further evidence that attachment is not a required signal for initiation of spore germination in $C$. graminicola. It was possible that Con A binding to the spore itself signaled germination to proceed. However, addition of Con A to spores on MW glass slides or in hanging drops did not induce germination significantly, except at the highest concentration, and then not to the same degree as on plastic coverslips.

As a further test of the role of surface hydrophobicity on spore germination, polystyrene sheeting was altered to produce different wettability characteristics by exposing it to UV light. This treatment causes polymer cross-linking and bond scission to occur (5), resulting in increased wettability of the polystyrene. The change in surface wettability, as indicated by $\theta_{\mathrm{A}}$, significantly affected spore germination rates. Interestingly, a very small change in surface hydrophobicity corresponded to a very large change in germination. This indicates the presence of a "threshold" effect of surface hydrophobicity on the induction of germination. The threshold in this experiment (between $54 \pm 5.5^{\circ}$ and $65 \pm 3.7^{\circ}$ ) agrees well with the threshold observed in the initial germination assays on a series of different surfaces (between 50 and $55^{\circ}$ ). Similar results were obtained for $P$. ampelicida on UV-altered polystyrene (15). The wettability values associated with a signaling threshold for germination in $C$. graminicola overlapped those reported for $P$. ampelicida.

In contrast to spore germination, appressorial induction appeared to be dependent on fungal attachment rather than surface hydrophobicity. Evidence for this was the efficient induction of appressoria on the hydrophilic surfaces of certain glass slides. These slides also produced significantly higher spore attachment values than would have been expected based on their surface wettabilities. Assuming that the degree of attachment of the germ tube is similar to that of the spore, this suggests that attachment is inductive for appressorial formation even if the attachment is not mediated by hydrophobic interactions. Additional evidence for the importance of attachment in appressorial induction was provided by the experiments with Con A: appressorial induction was almost entirely inhibited at a concentration of $1 \mathrm{mg} / \mathrm{ml}$ on plastic coverslips, which have a surface that is normally highly stimulatory for appressorial development. Spore attachment was also significantly inhibited at that concentration, although spore germination was unaffected. Spore attachment was also inhibited at lower concentrations of Con A, but to a lesser extent, and appressorial induction was relatively unaffected at those lower concentrations. This suggests that there is also a threshold of attachment that is sufficient to trigger optimal appressorial development.

This report provides the first evidence that a rigid, hydrophobic surface directly triggers spore germination in C. graminicola. The relationship between surface rigidity and hydrophobicity and spore germination is superseded by addition of nutrients, particularly carbon sources, to spore suspensions. This indicates that there are multiple response pathways involved in spore germination, and that this process in nature may be highly complex. If information on spore germination is to be useful in developing a control mechanism, we will need to seek out the convergence of the various signaling pathways and identify molecules that are critical to all of these pathways. These molecules will provide the best targets for chemical or genetic therapies designed to prevent spore germination in the field.

\section{ACKNOWLEDGMENTS}

These studies were supported in part by a grant from the office of the Vice Chancellor for Research and Graduate Studies at the University of Kentucky. This is publication 01-12-18 of the Kentucky Agricultural Experiment Station, published here with the approval of the director. We thank E. Nuckles and D. Brown for technical assistance; L. Penn and H. Huang of the University of Kentucky Department of Engineering, and E. Johnson of Lexmark International, for advice regarding contact angle measurements; and H. Hoch and B. Shaw for helpful discussions.

\section{LITERATURE CITED}

1. Bergstrom, G. C., and Nicholson, R. L. 1983. Microhumidity chamber for quantitative inoculation of attached corn leaves with fungal pathogens. Phytopathology 73:1040-1042.

2. Bergstrom, G. C., and Nicholson, R. L. 1999. The biology of corn anthracnose: Knowledge to exploit for improved management. Plant Dis. 83:596-608.

3. Braun, E. J., and Howard, R. J. 1994. Adhesion of Cochliobolus heterostrophus conidia and germlings to leaves and artificial surfaces. Exp. Mycol. 18:211-220.

4. Dean, R. A. 1997. Signal pathways and appressorium morphogenesis. Annu. Rev. Plant Pathol. 35:211-234.

5. Decker, C. 1989. Effect of UV radiation on polymers. Pages 541-608 in: Handbook of Polymer Science and Technology. Vol. 3. Applications and Processing Operations. N. P. Cheremisinoff, ed. Marcel Dekker, Inc., New York.

6. d'Enfert, C. 1997. Fungal spore germination: Insights from the molecular genetics of Aspergillus nidulans and Neurospora crassa. Fungal Genet. Biol. 21:163-172.

7. Egley, G. H. 1994. Substrate surface influences upon germination of Colletotrichum truncatum conidia. Can. J. Bot. 72:1758-1765.

8. Hamer, J. E., Howard, R. J., Chumley, F. G., and Valent, B. 1988. A mechanism for surface attachment in spores of a plant pathogenic fungus. Science 239:288-290.

9. Hughes, H. B., Carzaniga, R., Rawlings, S. L., Green, J. R., and O'Connell, R. J. 1999. Spore surface glycoproteins of Colletotrichum lindemuthianum are recognized by a monoclonal antibody which inhibits adhesion to polystyrene. Microbiology 145:1927-1936.

10. Jones, E. B. G. 1994. Fungal adhesion. Mycol. Res. 98:961-981.

11. Jones, M. J., and Epstein, L. 1989. Adhesion of Nectria haematococca macroconidia. Physiol. Mol. Plant Pathol. 35:453-461.

12. Jones, M. J., and Epstein, L. 1990. Adhesion of macroconidia to the plant surface and virulence of Nectria haematococca. Appl. Environ. Microbiol. 56:3772-3778.

13. Kennedy, J. F., Palva, P. M. G., Corella, M. T. S., Cavalcanti, M. S. M., and Coelho, L. C. B. B. 1995. Lectins, versatile proteins of recognition: 
A review. Carbohydr. Polym. 26:219-230.

14. Kim, Y.-K., Li, D., and Kolattukudy, P. 1998. Induction of $\mathrm{Ca}^{+}$calmodulin signaling by hard-surface contact primes Colletotrichum gloeosporioides conidia to germinate and form appressoria. J. Bacteriol. 180:5144-5150.

15. Kuo, K., and Hoch, H. C. 1996. Germination of Phyllosticta ampelicida pycnidiospores: Prerequisite of adhesion to the substratum and the relationship of substratum wettability. Fungal Genet. Biol. 20:18-29.

16. Kwon, Y. H., and Epstein, L. 1993. A $90 \mathrm{kDa}$ glycoprotein associated with adhesion of Nectria haematococca macroconidia to substrata. Mol. Plant-Microbe Interact. 6:481-487.

17. Kwon, Y. H., and Epstein, L. 1997. Isolation and composition of the 90 $\mathrm{kDa}$ glycoprotein associated with adhesion of Nectria haematococca macroconidia. Physiol. Mol. Plant Pathol. 51:63-74.

18. Kwon, Y. H., and Epstein, L. 1997. Involvement of the $90 \mathrm{kDa}$ glycoprotein in adhesion of Nectria haematococca macroconidia. Physiol. Mol. Plant Pathol. 51:287-303.

19. Leach, J., Lang, B. R., and Yoder, O. C. 1982. Methods for selection of mutants and in vitro culture of Cochliobolus heterostrophus. J. Gen. Microbiol. 128:1719-1729.

20. Leite, B., and Nicholson, R. L. 1992. Mycosporine-alanine: A selfinhibitor of germination from the conidial mucilage of Colletotrichum graminicola. Exp. Mycol. 16:76-86.

21. Mercure, E. W., Kunoh, H., and Nicholson, R. L. 1994. Adhesion of Colletotrichum graminicola conidia to corn leaves: A requirement for disease development. Physiol. Mol. Plant Pathol. 45:407-420.

22. Mercure, E. W., Kunoh, H., and Nicholson, R. L. 1995. Visualization of materials released from adhered, ungerminated conidia of Colletotrichum graminicola. Physiol. Mol. Plant Pathol. 46:121-135.

23. Mercure, E. W., Leite, B., and Nicholson, R. L. 1994. Adhesion of ungerminated conidia of Colletotrichum graminicola to artificial hydrophobic surfaces. Physiol. Mol. Plant Pathol. 45:421-440.

24. Moss, M. A., Sisken, B., Zimmer, S., and Anderson, K. W. 1999. Adhesion of nonmetastatic and highly metastatic breast cancer cells to endothelial cells exposed to shear stress. Biorheology 36:359-371.

25. Nicholson, R. L., and Epstein, L. 1991. Adhesion of fungi to the plant surface: Prerequisite for pathogenesis. Pages 3-23 in: The Fungal Spore and Disease Initiation in Plants and Animals. H. Hoch and G. Cole, eds. Plenum Press, New York.
26. Nicholson, R. L., and Warren, H. L. 1976. Criteria for evaluation of resistance to maize anthracnose. Phytopathology 66:86-90.

27. Panaccione, D. G., Vaillancourt, L. J., and Hanau, R. M. 1989. Conidial dimorphism in Colletotrichum graminicola. Mycologia 81:876-883.

28. Rasmussen, J. B., and Hanau, R. M. 1989. Exogenous scytalone restores appressorial melanization and pathogenicity in albino mutants of Colletotrichum graminicola. Can. J. Plant Pathol. 11:349-352.

29. Ritchie, S. W., Hanway, J. J., and Benson, G. O. 1993. How a Corn Plant Develops. J. C Herman, ed. Iowa State University of Science and Technology Cooperative Extension Service, Ames.

30. Schwartz, A. M. 1980. Contact angle hysteresis: A molecular interpretation. J. Colloid Interface Sci. 75:404-408.

31. Sela-Buurlage, M. B., Epstein, L., and Rodriguez, R. J. 1991. Adhesion of ungerminated Colletotrichum musae conidia. Physiol. Mol. Plant Pathol. 39:345-352.

32. Shaw, B. D., and Hoch, H. C. 1999. The pycnidiospore of Phyllosticta ampelicida: Surface properties involved in substratum attachment and germination. Mycol. Res. 103:915-924.

33. Shaw, B. D., and Hoch, H. C. 2000. $\mathrm{Ca}^{2+}$ regulation of Phyllosticta ampelicida pycnidiospore germination and appressorium formation. Fungal Genet. Biol. 31:43-53.

34. Takano, Y., Kikuchi, T., Kubo, Y., Hamer, J. E., Mise, K., and Furusawa, I. 2000. The Colletotrichum lagenarium MAP kinase gene CMK1 regulates diverse aspects of fungal pathogenesis. Mol. Plant-Microbe Interact. 13:374-383.

35. Terhune, B. T., and Hoch, H. C. 1993. Substrate hydrophobicity and adhesion of Uromyces urediospores and germlings. Exp. Mycol. 17:241-252.

36. Tuite, J. 1969. Plant Pathological Methods: Fungi and Bacteria. Burgess Publishing Company, Minneapolis, MN.

37. Warwar, V., and Dickman, M. B. 1996. Effects of calcium and calmodulin on spore germination and appressorium development in Colletotrichum trifolii. Appl. Environ. Microbiol. 62:74-79.

38. Webster, J., and Davey, R. A. 1984. Sigmoid conidial shape in aquatic fungi. Trans. Brit. Mycol. Soc. 83:43-52.

39. Young, D. H., and Kauss, H. 1984. Adhesion of Colletotrichum lindemuthianum spores to Phaseolus vulgaris hypocotyls and to polystyrene. Appl. Environ. Microbiol. 47:616-619.

40. Zettlemoyer, A. C. 1969. Hydrophobic surfaces. Pages 1-27 in: Hydrophobic Surfaces. F. M. Fowkes, ed. Academic Press, New York. 